\title{
Situación psicosocial de las adolescentes y toxemias del embarazo*
}

Jaime Salvador-Moysén, M. en C., ${ }^{(1)}$ Yolanda Martínez-López, M. en C., (2)

Angélica Lechuga-Q uiñones, Lic. en Psic., ${ }^{(1)}$ Rosario Ruiz-Astorga, Q uim. Farmacobiol., ${ }^{(1)}$

Alberto Terrones-González, Lic. en Psic. ${ }^{(1)}$

\section{Salvador-Moysén J, Martínez-López Y, Lechuga-Quiñones A, Ruiz-Astorga $R$, Terrones-González A. Situación psicosocial de las adolescentes y toxemias del embarazo. \\ Salud Publica Mex 2000;42:99-105.}

\section{Resumen}

Objetivo. Conocer la situación psicosocial percibida durante la gestación por dos grupos de adolescentes; uno de ellos, conformado por mujeres con diagnóstico de enfermedad hipertensiva inducida por el embarazo y, el otro, constituido por mujeres cualificadas como "sanas". Material y métodos Se eligió un diseño de casos y controles. De las ado lescentes gestantes que acudieron para la resolución de su embarazo al Hospital General de la ciudad de D urango, Durango, en los periodos de julio de 1996 a febrero de 1997, y de septiembre de 1998 a enero de 1999, se seleccionaron 39 casos y 88 controles de acuerdo con criterios explícitos del estudio, a quienes se les aplicó un cuestionario semiestructurado para la identificación de: estresores psicosociales, apoyo psicosocial, respuestas de enfrentamiento, intencionalidad, deseo y aceptación del embarazo, estado anímico y calidad de convivencia durante el mismo. Para el análisis de los datos, además de los procedimientos descriptivos, se estimó $\chi^{2}$ y la razón de momios con intervalos de confianza a 95\%. Resultados Las características sociodemográficas y ginecobstétricas de ambos grupos fueron similares; la percepción de fuentes de estrés fue mayor en el grupo de casos y la de fuentes de apoyo favoreció al grupo control, el estado anímico positivo y la convivencia satisfactoria durante el embarazo fueron referidos más frecuentemente con valo res estadísticamente significativos, por las integrantes del grupo control. Conclusiones. Se identificaron diferencias cualitativas en la percepción de los escenarios psicosociales por las mujeres participantes en el estudio, observándose una evaluación más favorable, estadísticamente significativa, por el grupo control.

Palabras clave:hipertensión; embarazo;adolescencia;México
Salvador-Moysén J, Martínez-López Y, Lechuga-Quiñones A, Ruiz-A storga $R$, Terrones-González A.

Psychosocial conditions of adolescents with toxemia of pregnancy.

Salud Publica Mex 2000;42:99-105.

\begin{abstract}
A bstract
Objective.To compare the perception of psychosocial conditions (during pregnancy) between two groups of adolescents; one group was integrated by yo ung wo men diagno sed with pregnancy induced-hypertension and the other group by clinically healthy women. Material and methods A case-control study design where 39 cases and 88 controls were chosen from pregnant adolescents seeking care at the General Hospital of Durango City, México, from July 1996 to February 1997, and from September 1998 to January 1999. Participants agreed to answer a semi-structured questionnaire to collect data on psychosocial support; psychosocial source of stress; coping responses; intention, desire and acceptance of pregnancy; and attitude and quality of socialization during pregnancy. Data analysis consisted of descriptive statistics, $\chi^{2}$ and odds ratios with $95 \%$ confidence intervals. Results. The two groups' sociodemographic and gynecoobstetric characteristics were similar. Perception of stressors was higher among cases; sources of support were greater among controls. Positive attitudes and satisfactory socialization during pregnancy were more frequently reported by controls, with statistically significant differences between groups. Conclusions We found qualitative differences in the perception of psychosocial conditions among study participants, with statistically significant favorable perceptions among controls.
\end{abstract}

Key words: hipertension; pregnancy; adolescence; Mexico

* Trabajo financiado parcialmente por el Sistema de Investigación Francisco Villa y el Consejo N acional de Ciencia y Tecnología, clave 9702076.

(1) Instituto de Investigación Científica. Universidad Juárez del Estado de Durango. Durango, México.

(2) Unidad de Epidemiología Clínica. Hospital General de Durango. Servicios de Salud de Durango. Durango, México.

Fecha de recibido: 14 de julio de 1999 •Fecha de aprobado: 16 de febrero de 2000

Solicitud de sobretiros: M. en C. Jaime Salvador Moysén. Instituto de Investigación Científica, Universidad Juárez del Estado de Durango. A venida Universidad y Fanny Anitúa s/n, 34000 Durango, Durango, México. 
L as toxemias del embarazo siguen representando en la actualidad, además de un relevante problema de salud pública ${ }^{1-6}$-caracterizado por su alta prevalencia en las mujeres gestantes de diferentes ámbitos geográficos y su impacto en la morbilidad y la mortalidad perinatal y materna- un reto para los investigadores biomédicos y clínicos, quienes no han dado, aún, una respuesta satisfactoria a la génesis de esta enfermedad.

El desconocimiento de la causa de este padecimiento, no obstante su carácter milenario, no implica la inexistencia de estudios cuyo objetivo principal haya sido el esclarecimiento de la trama causal. La cantidad de investigaciones realizadas con este propósito son extraordinariamente numerosas, y no obstante que se han identificado diversos factores de riesgo, así como condiciones fisiopatológicas y clínicas predictoras de la enfermedad, ${ }^{7-23}$ la realidad es que no existe un modelo explicativo satisfactorio a este problema de salud. Esto puede atribuirse en cierta medida a las estrategias que se han seleccionado para dar respuesta a las interrogantes relacionadas con su génesis, las cuales se han caracterizado fundamentalmente por un enfoque unicausal y biologicista, que poco puede aportar a la comprensión cabal de la enfermedad y de cómo se genera, no considerándose prácticamente las exigencias adaptativas de carácter psicosocial que surgen durante la gestación y que persisten hasta el final de la misma.

La importancia del entorno psicosocial en relación con el proceso salud-enfermedad se ha documentado en distintos estudios epidemiológicos sobre diversos problemas de salud, ${ }^{24-27}$ y los planteamientos hipotéticos para explicar esta asociación postulan que la situación psicosocial interviene como un factor condicionante inespecífico que facilita o inhibe la expresión de factores de riesgo específicos, teniendo un carácter protector cuando se evalúa como favorable o positiva, y representando un riesgo cuando se califica como desfavorable. Esta situación psicosocial está conformada, de acuerdo con $\mathrm{Cassel}^{28}$ por dos tipos de condiciones: en primer lugar, por aquellas que los individuos perciben como favorables y que son denominadas apoyo psicosocial y, en segundo lugar, la condición que corresponde a los eventos que se perciben como desfavorables y se les denomina estresores psicosociales. Tanto los estresores como el apoyo psicosocial constituyen elementos que coexisten en la experiencia cotidiana de todas las personas, y se propone que la situación psicosocial resultante (favorable o desfavorable) es una consecuencia relacionada directamente con la condición psicosocial prevaleciente (estresante o de apoyo). Desde esta perspectiva, el estrés psicosocial se considera el resultado de la relación que se establece entre la persona y su ambiente en el contexto de un proceso multivariado con específicas consecuencias en el proceso salud-enfermedad, el estresor está representado por aquellas situaciones familiares, laborales, económicas, sociales y culturales que demandan y exceden los recursos personales de los individuos, de tal modo que significan amenaza, daño o desafío; mediante el apoyo psicosocial, la persona obtiene satisfacción de sus necesidades de reconocimiento social, afectivas, de comunicación, de solidaridad y económicas. ${ }^{28-31}$ La conceptualización del estrés, como un proceso mediador psicobiológico, da sustento a las propuestas tanto teóricas como empíricas que sugieren una asociación de las condiciones del entorno psicosocial, con la expresión clínica de distintas enfermedades. ${ }^{32,33}$

Metodológicamente, el modelo epidemiológico constituye una alternativa satisfactoria para conocer las relaciones existentes de un conjunto de variables psicosociales con la enfermedad hipertensiva inducida por el embarazo. Este modelo también permite explorar y analizar la relación de estos indicadores psicosociales con otros de naturaleza clínica y bioquímica, de tal forma que hace factible el diseño de nuevas estrategias para aclarar los procesos cruciales del entramado causal de este problema. El propósito del presente estudio es evaluar de manera metódica y rigurosa la situación psicosocial de dos grupos de adolescentes embarazadas, y estimar su asociación con las toxemias del embarazo. El primer grupo se conformó por pacientes en las cuales se estableció el diagnóstico de enfermedad hipertensiva inducida por el embarazo, y el segundo fue constituido por adolescentes embarazadas, quienes se cualificaron como sanas de acuerdo a criterios explícitos de la investigación. Los fundamentos conceptuales del trabajo se sustentan en las aportaciones de Cassel, Milsum y Selye, ${ }^{28,32,34}$ operativamente la situación psicosocial se consideró como la resultante de la interacción del apoyo psicosocial (representado por las fuentes de apoyo en los niveles conyugal, familiar y social) y de los estresores psicosociales (conformado por aquellas situaciones estresantes que se generaron en los ámbitos conyugal, familiar y social durante el proceso gestacional), la medición de estos indicadores se realizó a través de la aplicación de un instrumento diseñado exprofeso.

\section{Material y métodos}

Se utilizó un diseño de casos y controles. Se entrevistaron 127 mujeres adolescentes quienes acudieron para la resolución de su embarazo al Hospital General de la ciudad de Durango, Durango, en los periodos com- 
prendidos de entre julio de 1996 a febrero de 1997, y de septiembre de 1998 a enero de 1999. De ellas, 39 tuvieron el diagnóstico de enfermedad hipertensiva inducida por el embarazo y las restantes 88 conformaron el grupo control. El tamaño muestral se estimó con la siguiente fórmula: ${ }^{35}$

$$
\mathrm{P}=\frac{(\mathrm{Pco}+\mathrm{Pca})}{2}
$$

Pco $=$ estimación de la proporción de controles que estuvieron expuestos

$\mathrm{Pca}=$ estimación de la proporción de casos que estuvieron expuestos

$$
\mathrm{N}=\frac{2(\mathrm{Z} \alpha+Z \beta)^{2} p(1-p)}{(\text { Pco }- \text { Pca })^{2}}
$$

donde:

$$
\mathrm{N}=\frac{2(1.65+0.84)^{2} 0.23(0.77)}{(0.11-0.35)^{2}}=38
$$

Para los valores de zeta se seleccionó una $\alpha=0.05$ y una $\beta=$ 0.20

Definición de caso. Se consideró como tal a la adolescente (20 años de edad o menos) en quien se estableció el diagnóstico de enfermedad hipertensiva inducida por el embarazo, por personal médico del servicio de ginecobstetricia del Hospital General de la ciudad de Durango, Durango, de la Secretaría de Salud. Definición de control ("sanas"). Se definió así a la adolescente embarazada, atendida en la misma institución y en el mismo periodo que los casos, que durante la gestación cumplió con los siguientes criterios:

- No haber sido diagnosticada como hipertensa previamente o al inicio de la gestación

- No haber presentado algún proceso patológico durante el embarazo que hubiera ameritado hospitalización

- La evaluación de APGAR del producto de la gestación debió ser ocho a los cinco minutos de haber nacido y no haber presentado malformaciones externas.

Se elaboró un cuestionario semiestructurado para la identificación de estresores psicosociales, apoyo psicosocial y respuestas de enfrentamiento, ${ }^{30,36-39}$ que las participantes hayan percibido durante la gestación en las dimensiones conyugal, familiar y social. La dimensión conyugal involucra al padre del niño, indepen- dientemente del carácter formal de la relación con la madre adolescente; la familiar, está constituida por el padre, madre y hermanos(as), y la social, por las amigas(os), escuela o trabajo de las integrantes de los grupos estudiados.

La evaluación de la situación psicosocial se realizó de la siguiente manera: a la sumatoria del puntaje obtenido en el apartado de apoyo psicosocial se le restó el observado en el de estresores psicosociales. La presencia de estresores se calificó negativamente y la de apoyo positivamente. La ausencia de uno o de otro se calificó con 0 . Los valores que se otorgaron a los integrantes del contexto psicosocial fueron los siguientes: padre, madre y compañero 1.5 puntos; hermanos(as) y otros familiares 1.0 punto y, finalmente, amigas(os), escuela, trabajo y otros 0.5 puntos. De acuerdo con el puntaje final se formaron cuatro grupos; el negativo, cuando la sumatoria fue igual o menor a 0 ; el levemente positivo, cuando los valores fueron de 0.5 a 1.5; el moderadamente positivo, si la sumatoria fue de 2.0 a 4.5 puntos, y el fuertemente positivo, cuando el puntaje fue de 5.0 a 7.0 puntos.

Las opciones de respuesta del cuestionario aplicado no sólo permitieron registrar la existencia o ausencia de los indicadores psicosociales, sino también el carácter cualitativo de los mismos. El instrumento también contiene reactivos para el registro de datos sociodemográficos y clínicos, así como una breve sección que permite conocer la intencionalidad, deseo, aceptación del embarazo, estado anímico y calidad de convivencia durante el mismo. Este cuestionario se fundamentó, conceptual y metodológicamente, en un constructo desarrollado y validado por GonzálezForteza. ${ }^{31-40}$

Tanto los casos como los controles se identificaron en expedientes clínicos y registros del Hospital General de la ciudad de Durango, Durango. Las entrevistas fueron efectuadas en dos periodos, el primero de ellos comprendido de julio de 1996 a febrero de 1997 y el segundo de septiembre de 1998 a enero de 1999. La mayoría de las participantes fueron entrevistados en su domicilio y una pequeña proporción en el propio hospital (el periodo transcurrido entre la resolución del embarazo y la entrevista fue en promedio de 2.0 meses para las integrantes del primer periodo y de 4.6 meses para las observaciones del segundo; los resultados que se presentan corresponden al análisis de todos los datos obtenidos). Las actividades de búsqueda en los expedientes clínicos y archivos hospitalarios así como la aplicación de los cuestionarios se efectuaron por dos equipos debidamente entrenados. Se realizaron procedimientos de uniformidad en la técnica de entrevista y se efectuó una prueba piloto. El 
primero de los equipos estuvo formado por cuatro estudiantes de la licenciatura de medicina, una estudiante de psicología (en prácticas de servicio social), y uno de los responsables del proyecto; el segundo equipo se integró por una licenciada en psicología y dos licenciadas en enfermería. Para la realización de las entrevistas se solicitó el consentimiento de las participantes, y se señaló el carácter anónimo y confidencial de la información.

La caracterización sociodemográfica, clínica (ginecobstétrica) y psicosocial, se realizó mediante la aplicación de procedimientos estadísticos descriptivos. La $\chi^{2}$, la estimación de la razón de momios y sus intervalos de confianza a $95 \%$, se emplearon para evaluar las fuentes de estrés y de apoyo en el contexto familiar, la interacción apoyo-estresores en las diferentes dimensiones analizadas, así como el estado anímico y calidad de convivencia durante la gestación.

\section{Resultados}

La edad promedio de las integrantes del grupo de casos fue de 18.3 años con una desviación estándar de 1.5 , y la del grupo control, de 17.6 \pm 1.2 años. Con referencia a las variables sociodemográficas y ginecobstétricas, en el cuadro I se observa que el estado civil prevaleciente es el de unidas en los dos grupos estudiados; en cuanto a escolaridad más de $80 \%$ de ambos grupos refirió nueve años o menos de estudio; la ocupación de ama de casa fue referida por $92 \%$ de las integrantes del grupo de casos y $86 \%$, de los controles; la condición de primigestas se observó en más de $70 \%$ de ambos grupos y el número de asistencias a consultas de control prenatal fue superior en el grupo control, aunque las diferencias no fueron estadísticamente significativas.

En el cuadro II se observan los resultados obtenidos al realizar el contraste de casos y controles con relación al número de integrantes del grupo familiar que representaron una fuente de estrés o de apoyo psicosocial. El denominador poblacional se estimó considerando el total de familiares (exclusivamente padre, madre y hermanos) que convivieron con cada una de las embarazadas que conformaron ambos grupos de estudio. Se observa una mayor frecuencia de fuentes de estrés en el grupo de casos al compararse con el grupo control, los valores obtenidos tienen significancia estadística; la evaluación de las fuentes de apoyo arroja valores estadísticamente significativos con relación a la ausencia de apoyo durante la gestación, condición que fue referida con más frecuencia por el grupo de casos.
Cuadro I

Características sociodemográficas

Y GINECOBSTÉTRICAS DE ADOLESCENTES EMBARAZADAS.

Hospital General de la ciudad de Durango. Durango, MÉxico, 1996-1999

Variable $\quad \frac{\text { Casos }}{n=39} \quad \frac{\text { Controles }}{n=88 \%}$

\begin{tabular}{ccccc}
$\begin{array}{c}\text { Estado civil } \\
\text { Soltera }\end{array}$ & 12 & 31 & 29 & 33 \\
\hline Unida & 27 & 69 & 59 & 67
\end{tabular}

\begin{tabular}{lrrrr}
$\begin{array}{c}\text { Escolaridad } \\
\leq 6 \text { años }\end{array}$ & 16 & 41 & 37 & 42 \\
\hline $7-9$ años & 16 & 41 & 41 & 47 \\
\hline$\geq 10$ años & 6 & 15 & 8 & 9 \\
\hline Se desconoce & 1 & 3 & 2 & 2
\end{tabular}

0 cupación

\begin{tabular}{lrrrr} 
Ama de casa & 36 & 92 & 76 & 86 \\
\hline Empleada & 2 & 5 & 11 & 13 \\
\hline Estudiante & 1 & 3 & 1 & 1 \\
\hline Total & 39 & 100 & 88 & 100
\end{tabular}

Paridad

\begin{tabular}{lrrrr} 
Primigestas & 29 & 74 & 70 & 80 \\
\hline Secundigestas & 8 & 20 & 13 & 15 \\
\hline$>2$ gestaciones & 1 & 3 & 3 & 3 \\
\hline Se desconoce & 1 & 3 & 2 & 2
\end{tabular}

Control prenatal

\begin{tabular}{lrrrr} 
Sin control & 8 & 20 & 14 & 16 \\
\hline$\leq 3$ consultas & 11 & 28 & 15 & 17 \\
\hline $4-5$ consultas & 7 & 18 & 14 & 16 \\
\hline$\geq 6$ consultas & 12 & 31 & 42 & 48 \\
\hline Se desconoce & 1 & 3 & 3 & 3
\end{tabular}

En el cuadro III se describe el puntaje resultante de la interacción apoyo-estresores en los dos grupos estudiados, puntaje del que se derivan los distintos estratos de la situación psicosocial; es clara la diferencia en las categorías extremas, observándose en la negativa (hasta 0 puntos) una frecuencia mayor en las adolescentes que conformaron los casos, la situación opuesta se aprecia en la categoría "fuertemente positiva" (5.0 -7.0 puntos) en la cual se ubicó una mayor proporción de las integrantes del grupo control. La comparación mostró una diferencia estadísticamente significativa $\left(\chi^{2}, p<0.05 ; \mathrm{RM}=4.14, \mathrm{IC} 95 \% 1.07-15.9\right)$. El 
Cuadro II

NúMERO DE INTEGRANTES DEL GRUPO FAMILIAR QUE SIGNIFICARON FUENTES DE ESTRÉS O DE APOYO entre adolescentes. Hospital General de la Ciudad de Durango. Durango, México, 1996-1999

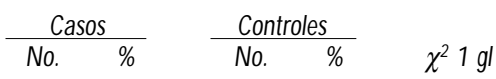

Fuente de estrés

\begin{tabular}{lrrrrc} 
Presente & $25 / 100$ & 25 & $27 / 230$ & 12 & $p<0.01$ \\
\hline Ausente & $75 / 100$ & 75 & $203 / 230$ & 88 & ns
\end{tabular}

Fuente de apoyo

\begin{tabular}{lrrrrc} 
Presente & $75 / 100$ & 75 & $196 / 230$ & 85 & ns \\
\hline Ausente & $25 / 100$ & 25 & $34 / 230$ & 15 & $p<0.05$
\end{tabular}

Fuente: Instrumento de riesgo psicosocial ns: no significativa

\section{Cuadro III \\ Estratos de PERCEPCIÓN DE LA SITUACIÓN PSICOSOCIAL ENTRE ADOLESCENTES EMBARAZADAS. Hospital General de la ciudad de Durango. Durango, Méxıco, 1996-1997}

\begin{tabular}{lrrrrr}
\multirow{2}{*}{ Puntaje } & \multicolumn{2}{c}{ Casos } & & \multicolumn{2}{c}{ Controles } \\
\cline { 2 - 3 } \cline { 5 - 6 } Ho. & $\%$ & & No. & $\%$ \\
\hline $0.5-1.5$ & 6 & 15 & & 6 & 7 \\
\hline $2.0-4.5$ & 21 & 54 & 44 & 50 \\
\hline $5.0-7.0$ & 7 & 18 & 29 & 33 \\
\hline Total & 39 & 100 & 88 & 100
\end{tabular}

Fuente: Instrumento de riesgo psicosocial

análisis de los datos relacionados con la presencia de estado anímico positivo y convivencia satisfactoria durante el embarazo mostró una frecuencia de 55\% $(48 / 88)$ en el grupo control vs. $28 \%$ (11/39) en el de casos, con una diferencia estadísticamente significativa $\left(\chi^{2}, 7.56\right) ; p<0.01 ; \mathrm{RM}, 3.05$; IC95\%1.37-6.76; estos resultados permiten inferir, conjuntamente con los indicadores ya referidos (estresores y soporte), la percepción de escenarios psicosociales distintos por las adolescentes entrevistadas, observándose que el grupo de "sanas" mostró una condición más favorable en las diferentes dimensiones exploradas.

\section{Discusión}

Los resultados obtenidos en este trabajo permiten identificar diferencias en la situación psicosocial del grupo de casos respecto de los controles. Las diferencias se observaron tanto en la evaluación independiente de estresores y de apoyo, como en el análisis de la situación psicosocial (producto de la interacción apoyoestresores), esta última representa la medición empírica más sensible, dado que evalúa la resultante de la percepción global de los indicadores psicosociales de interés. La interpretación de los datos se realizó desde un enfoque conceptual, sustentado fundamentalmente en las aportaciones de Cassel, Milsum y Selye, ${ }^{28,32,34} \mathrm{y}$ puede resumirse de la siguiente manera: las diferencias observadas, con una significancia estadística, en la percepción de las condiciones del entorno psicosocial, tanto en el grupo de casos como en el de controles, sugieren una asociación de la situación psicosocial desfavorable con la enfermedad hipertensiva inducida por el embarazo.

La selección de los grupos en lo referente a las características sociodemográficas y ginecobstétricas permitió su comparabilidad y el control de estas variables, las cuales son potencialmente confusoras. Los resultados de este estudio son congruentes con las hipótesis referidas en la literatura acerca de la importancia de las variables psicosociales como un elemento que condiciona la expresión de factores de riesgo específicos, $28,29,32-34,38,41,42$ es importante referir que se han desarrollado investigaciones que han buscado e identificado una asociación de estrés laboral con la hipertensión inducida por el embarazo y preeclampsia; ${ }^{43-45}$ la diferencia conceptual y metodológica más importante de estos trabajos con el que aquí se presenta, descansa en el hecho de que en este último la exploración se realizó en diferentes dimensiones del contexto psicosocial a diferencia de los primeros en los cuales se analiza de manera exclusiva la dimensión laboral. La réplica del estudio en otros ámbitos socioculturales permitirá saber si las conclusiones obtenidas, de carácter preliminar, pueden generalizarse; si la respuesta es afirmativa se posibilita el avance del análisis del problema en sus distintos niveles de organización a partir de nuevas interrogantes, por ejemplo: ¿Existe algún indicador bioquímico que se asocie directamente con el balance de la situación psicosocial de la adolescente embarazada? Y si existiese este indicador: ¿Cuál es su rol en la expresión sintomática de las toxemias del embarazo? ¿Cómo se asocia con los distintos factores de riesgo validados en numerosas investigaciones? No obstante la complejidad del problema ana- 
lizado, tal vez el replanteamiento de viejas preguntas y el empleo de modelos con una concepción interdisciplinaria que hagan vigente el concepto de plausibilidad y busquen la articulación lógica de las observaciones bioquímicas, clínicas y psicosocioepidemiológicas permitan identificar los procesos subyacentes cruciales de la enfermedad y encontrar no sólo su explicación, sino una nueva forma de mirar la investigación del proceso salud enfermedad.

\section{Agradecimientos}

Los autores agradecen a la doctora Catalina GonzálezForteza su valiosa colaboración para el desarrollo del instrumento de riesgo psicosocial utilizado en el estudio, también agradecen a las licenciadas en enfermería María Eva Ortiz Cisneros y Martha B. Ortega Nevárez su participación en la aplicación de los cuestionarios.

\section{Referencias}

1. López-Llera M. La toxemia del embarazo. 2ạedición.México, D.F.: Editorial Limusa,1985:53-66.

2. Reyes S. Mortalidad materna en México, México, D.F.: Instituto Mexicano del Seguro Social,1994:28-41.

3. Saftlas A, O Ison D, Franks A, A trash H, Pokras R. Epidemiology of preclampsia and eclampsia in the U nited States, 1979-1986.Am J O bstet Gynecol 1990;163:460-465.

4. Davey D, MacGillivray I. The classification and definition of the hypertensive disorders of pregnancy. Am J O bstet Gynecol 1988;158:892-898. 5. Zhang J, Zeister J, Hatch M, Berkowitz G. Epidemiology of pregnancyinduced hypertension. Epidemiol Rev 1997;19:218-232.

6. Wergeland $E$, Strand $K$.W orking conditions and prevalence of pre-eclampsia, N orway 1989. Int J Gynecol 0 bstet 1997;58:189-196.

7. Morris $\mathrm{N}, \mathrm{O}$ sborn $\mathrm{S}, \mathrm{W}$ right $\mathrm{H}, \mathrm{H}$ art $\mathrm{A}$. Effective uterine blood-flow during exercise in normal and pre-eclamptic pregnancies. Lancet 1956: 481-484.

8. Fish J, Bartholomew R, Colvin E, G rimes W, Lester W, Galloway W. The relationship of pregnancy weighth gain to toxemia. Am J O bstet Gynecol 1959;78:743-754.

9. Castelazo L, Karchmer S, Chávez J, Shor V, O ntiveros E. N utrición y estado grávido puerperal. IV. Papel de la nutrición materna en la etiología de la toxemia gravídica. Ginecol 0 bstet Mex 1967;22:469-482.

10. Condie R, 0 gston D. Sequential studies on components of the haemostatic mechanism in pregnancy with particular reference to the development of pre-eclampsia. Br J O bstet Gynecol 1976;83:938-942.

11. Frydman R, Belaisch-Allart J, Fries N, H azout A, G lissant A, Testart J.An obstetric assessment of the first 100 births from the in vitro fertilization program at Clamart, France. Am J O bstet Gynecol 1986;154:550-555.

12. Seang-Lin Tan, D oyle P, Campbell S, Beral V, Rizk B, Brinsden P et al. O bstetric outcome of in vitro fertilization pregnancies compared with normally conceived pregnancies.Am J O bstet Gynecol 1992;167:778-784. 13. Belizán J,Villar J, Repke J.The relationship between calcium intake and pregnancy-induced hypertension:U p-to-date evidence.Am J 0 bstet Gynecol 1988;158:898-902.
14. Pinto A, Sorrentino R, Sorrentino P, Guerritore T, Miranda L, Biondi A et al. Endothelial derived relaxing factor released by endothelial cells of human umbilical vessels and its impairment im pregnancy-induced hypertension. Am J O bstet Gynecol 1991;164:507-513.

15. Eskenazi B, Fenster L, Sydney S.A multivariate analysis of risk factors for preeclampsia. JAMA 1991;266:237-241.

16. MCC arthy A, W oolfson R, Raju S, Poston L. Abnormal endothelial cell function of resistance arteries from women with preeclampsia.Am J 0 bstet Gynecol 1993;168:1323-1330.

17. Sibai B, El N azer A, G onzález-Ruiz A. Severe preeclampsia-eclampsia in young primigravid women: Subsequent pregnancy outcome and remote prognosis. Am J O bstet Gynecol 1986;155:1011-1016.

18. Combs A, Katz M, Kitzmiller J, Brescia R. Experimental preeclampsia produced by chronic constriction of the lower aorta: Validation with longitudinal blood pressure measurements in conscious Rhesus monkeys.Am J O bstet Gynecol 1993;169:215-223.

19.Y ves Robillard P, Hulsey Th, Périanin J, Janky E, Habib-Miri E, Papiernik E.Association of pregnancy-induced hypertension with duration of sexual cohabitation before conception. Lancet 1994;344:973-975.

20. Cincotta R, Brennecke S. Family history of pre-eclampsia as a predictor for pre-eclampsia in primigravidas. Int J Gynecol O bstet 1998;60: 23-27.

21. Repke J, Robinson J.The prevention of pre-eclampsia and eclampsia. Int I Gynecol O bstet 1998;62:1-9.

22. Shaarawy M,Aref A, Salem E, Sheiba M. Radical-scavening antioxidantts in pre-eclampsia and eclampsia. Int J Gynecol 0 bstet 1998;60:123-128.

23. Sagol S, Ö zkinay E, O zsener S. Impaired antioxidant activity in women with pre-eclampsia. Int J Gynecol 0 bstet 1999;64:121-127.

24. N uckolls K, C assel J, Kaplan B. Psychosocial assets, life crisis and the prognosis of pregnancy.Am J Epidemiol 1972;95:431-441.

25. Berkman L, Syme L. Social networks, host resistance and mortality: A nine year follow-up study of Alameda county residents. Am J Epidemiol 1979;109:186-204.

26. Kaplan G, C amacho T. Perceived health and mortality:A nine year folowup of the human population laboratory cohort. Am J Epidemiol 1983; 117:292-304.

27. Stone A, Mezzacappa E, D onatone B, Gonder M. Psychosocial stress and social support are associated with prostate-specific antigen levels in men: Results from a community screening program. Health Psychol 1999:18:482-486

28. Cassel J. Psychosocial processes and "stress" :Theoretical formulation. Int J Health Serv 1974;4:471-482.

29. Mouriño R, Viniegra L. Diseño y validación de un instrumento para valorar el entorno psicosocial. Salud Publica Mex 1991;33:38-48

30. Lazarus R. Theory-based stress measurement. Psychol Inquiry 1990; 1:3-13.

31. González-Forteza C, Salgado V, Rodríguez E. Estresores cotidianos y su relación con el malestar emocional en adolescentes mexicanos. Psicopatología (Madrid) 1995;15:8-11.

32. Milsum J.A model of the eustress system for healh and illness. Behav Sci 1985;30:179-186.

33. Peiró JM, Salvador A. Control del estrés laboral. Madrid: Eudema,1993:53-67.

34. Selye H.The evolution of the stress concept. Am J Cardiol 1970;26: 289-299.

35. Instituto de Investigación Científica. La propuesta de investigación y el muestreo. Durango, D go.: Secretaría de Educación Cultura y DeporteUniversidad Juárez del Estado de Durango, 1995:61-95.

36. Pearlin L, Schooler C. The structure of coping. J Health Social Behav 1978:19:2-21

37. Pearlin L, Menaghan E, Lieberman M, Mullan J.The stress process. J Health Social Behav 1981;22:337-356

38. Cervantes R, Castro F. Stress, coping, and mexican american mental health:A systematic review. Hispanic J Behav Sci 1985;7:1-73. 
39. 0 mar AG. Stress y coping. Las estrategias de coping y sus interrelaciones con los niveles biológico y psicológico. Buenos Aires: Lumen,1995: 61-79.

40. González-Forteza C. Estrés psicosocial y respuestas de enfrentamiento: impacto sobre el estado emocional en adolescentes (tesis de maestría). México, D.F.: UN AM,1992.

41. Viniegra L. ¿Q ué significa la resolución de un problema clínico? Rev Invest Clin 1981;33:151-159.

42. Salvador J, De la Luna E, Reid A, Rivera L. Un enfoque bio-psico-social de la enfermedad hipertensiva aguda del embarazo. Salud Publica Mex 1989;31:757-762.
43. Klonoff-C ohen H, Cross J, Pieper C . Job stress and preeclampsia. Epidemiology 1996;7:245-249.

44. Landsbergis P, Hatch M. Psychosocial work stress and pregnancy-induced hypertension. Epidemiology 1996;7:346-351.

45. Klebanoff M, Shiono P, Rhoads G. 0 utcomes of pregnancy in a national sample of resident physicians. N Engl J Med 1990;323:1040-1045. 